Pesq. Vet. Bras. 38(5):973-980, maio 2018

\title{
Caracterização do gênero, da raça e da idade de uma população de 7.780 cães da Região Central do Rio Grande do Sul submetidos à necropsia ao longo de cinco décadas $(1964-2013)^{1}$
}

\author{
Mariana M. Flores ${ }^{2 *}$, Renata D. Mazaro ${ }^{3}$, Ana Paula S. Poeta ${ }^{4}$, Glaucia D. Kommers ${ }^{5}$ \\ e Rafael A. Fighera ${ }^{5}$
}

\begin{abstract}
Flores M.M., Mazaro R.D., Poeta A.P.S., Kommers G.D. \& Fighera R.A. 2018. [Characterization of gender, breed and age in a population of 7,780 dogs submitted for necropsy over five decades (1964-2013) in central Rio Grande do Sul, Brazil.] Caracterização do gênero, da raça e da idade de uma população de 7.780 cães da Região Central do Rio Grande do Sul submetidos à necropsia ao longo de cinco décadas (1964-2013). Pesquisa Veterinária Brasileira 38(5):973-980. Departamento de Patologia, Universidade Federal de Santa Maria, Av. Roraima 1000, Santa Maria, RS 97105-900, Brazil. E-mail: anemiaveterinaria@yahoo.com.br

Based on the lack of demographic database on the canine population living in the midland region of Rio Grande do Sul State, Brazil, and on the need for a "control population" in the accomplishment of several prevalence-based studies of different diseases diagnosed at the Laboratório de Patologia Veterinária (LPV) of the Universidade Federal de Santa Maria (UFSM), the objective of this study was to describe the breed, gender and age characteristics of the population of dogs necropsied in this diagnostic service over 50 years (1964-2013). The protocols of necropsies performed on dogs and recorded in the LPV-UFSM between 1964 and 2013 were reviewed, and information related to gender, age and breed from dogs from all the districts within the Central Region of RS were separated. A total of 7,780 dogs were necropsied, among which 469 (6\%) were registered in the first decade (1964-1973), $1,133(14.6 \%)$ in the second decade (1974-1983), 1,334 (17.1\%) in the third decade (1984-1993), 1,705 (22\%) in the fourth decade (1994-2003) and 3,139 (40.3\%) in the fifth decade (2004-2013). Of dogs whose gender was reported in the protocols, $52.6 \%$ were males and $47.4 \%$ were females. The median age of death was 3 years. Of dogs whose breed was reported in the protocols, $59.8 \%$ were purebred and $40.2 \%$ were mixed breed. The most common large and giant breed dogs were German Shepherd Dog (17.2\%), Boxer (6.9\%), Rottweiler (5.3\%), Fila Brasileiro (4.6\%), English Pointer (3.9\%), Collie Rough (3.7\%), Dobermann (3.7\%), and Labrador Retriever (2.1\%). The most common small and medium breed dogs were Poodle (8.9\%), Dachshund (6.3\%), Miniature Pinscher (5.6\%), English Cocker Spaniel (4.5\%), Pekingese (3.4\%), Yorkshire Terrier (3.3\%), and Brazilian Terrier $(2.8 \%)$. The percentage of females and the median age of death showed an increase during the five decades of this study. Although there has been a significant increase in the proportion
\end{abstract}

\footnotetext{
${ }^{1}$ Recebido em 16 de fevereiro de 2017.

Aceito para publicação em 26 de março de 2017.

Parte da Tese de Doutorado do primeiro autor.

${ }^{2}$ Programa de Pós-Graduação em Medicina Veterinária, área de concentração em Patologia e Patologia Clínica Veterinária, Centro de Ciências Rurais (CCR), Universidade Federal de Santa Maria (UFSM), Av. Roraima 1000, Bairro Camobi, Santa Maria, RS 97105-900, Brasil. *Autor para correspondência: marianamflores@yahoo.com.br

${ }^{3}$ Curso de Medicina Veterinária, Centro de Ciências Rurais (CCR), Universidade Federal de Santa Maria (UFSM), Av. Roraima 1000, Bairro Camobi, Santa Maria, RS 97105-900. Bolsista PIBIC/CNPq/UFSM. E-mail: renatamazaro92@gmail.com
}

\footnotetext{
${ }^{4}$ Laboratório de Epidemiologia Veterinária (EPILAB), Departamento de Medicina Veterinária Preventiva, Faculdade de Medicina Veterinária, Universidade Federal do Rio Grande do Sul (UFRGS), Av. Bento Gonçalves 9090, Porto Alegre, RS 95320-000, Brasil. E-mail: paulaserafinipoeta@gmail.com

${ }^{5}$ Laboratório de Patologia Veterinária (LPV), Departamento de Patologia, Universidade Federal de Santa Maria (UFSM), Av. Roraima 1000, Bairro Camobi, Santa Maria, RS 97105-900, Brasil.E-mails: glaukommers@yahoo. com, Rafael Fighera: anemiaveterinaria@yahoo.com.br
} 
of pure breed dogs compared to mongrels, we observed some changes in the occurrence of different breeds over time, including a significant decrease in the frequency of the Pekingese, Brazilian Terrier, English Pointer, and German Shepherd Dog, and significant increase in the frequency of the Poodle, Dachshund, and Labrador Retriever. The results presented here will serve as an allowance for future comparative studies of disease prevalence in dogs of Central Region of RS, helping to a more correct understanding and interpretation of results from these data surveys.

INDEX TERMS: Gender, breed, age, dogs, necropsy, demographic study, data base, data survey, canine population, mortality.

RESUMO-- Devido à ausência de um banco de dados demográficos da população canina que habita a Região Central do Rio Grande do Sul (RS) e à necessidade em se estabelecer uma "população controle" para a melhor interpretação da prevalência das doenças diagnosticadas pelo Laboratório de Patologia Veterinária (LPV-UFSM) da Universidade Federal de Santa Maria (UFSM), este estudo objetivou realizar uma análise das características relacionadas à raça, ao gênero e à idade dos cães necropsiados neste serviço de diagnóstico ao longo de 50 anos (1964-2013). Para isto, os laudos de necropsias de cães, realizadas entre 1964 e 2013, foram revisados, e deles foram retiradas informações referentes ao gênero, à idade e às raças de todos os cães oriundos dos municípios que compõem a Região Central do RS. Ao todo, 7.780 cães foram necropsiados; desses, 469 (6\%) na primeira década (1964-1973), 1.133 $(14,6 \%)$ na segunda década (1974-1983), $1.334(17,1 \%)$ na terceira década (1984-1993), 1.705 (22\%) na quarta década (1994-2003) e $3.139(40,3 \%)$ na quinta década (2004-2013). Do total de cães com gênero informado nos laudos, $52,6 \%$ eram machos e 47,4\% eram fêmeas. A mediana da idade de morte foi de três anos. Dos cães cuja raça foi informada nos laudos, 59,8\% eram de raça definida (RD) e 40,2\% não tinham raça definida (SRD). As raças de porte grande ou gigante mais frequentes foram: Pastor Alemão $(17,2 \%)$, Boxer $(6,9 \%)$, Rottweiler $(5,3 \%)$, Fila Brasileiro $(4,6 \%)$, Pointer Inglês (3,9\%), Collie Pelo Longo (3,7\%) Dobermann $(3,7 \%)$ e Labrador Retriever $(2,1 \%)$. As raças de porte pequeno ou médio mais frequentes foram: Poodle $(8,9 \%)$, Dachshund $(6,3 \%)$, Pinscher Miniatura (5,6\%), Cocker Spaniel Inglês $(4,5 \%)$, Pequinês $(3,4 \%)$, Yorkshire Terrier $(3,3 \%)$ e Terrier Brasileiro (2,8\%). Houve um aumento na proporção de fêmeas e um crescimento na mediana referente à idade de morte ao longo das cinco décadas avaliadas. Apesar de não ter havido um aumento relevante na proporção de cães de RD em comparação com os SRD, observaram-se algumas mudanças na ocorrência de diferentes raças ao longo do tempo, incluindo principalmente uma dramática diminuição na percentagem de Pequinês, Terrier Brasileiro, Pointer Inglês e Pastor Alemão, e um aumento marcado na percentagem de Poodle, Dachshund, Rottweiler e Labrador Retriever. Os resultados aqui apresentados servirão como um subsídio comparativo para futuros estudos retrospectivos sobre prevalência de doenças em cães da Região Central do RS, auxiliando para uma mais correta compreensão e interpretação dos resultados encontrados nesses levantamentos de dados.

TERMOS DE INDEXAÇÃO: Gênero, raça, idade, população, cães, Rio Grande do Sul, necropsia, estudo demográfico, base de dados, levantamento de dados, população canina, mortalidade.

\section{INTRODUÇÃO}

Estudar os aspectos epidemiológicos de uma determinada doença exige que se conheçam as características do grupo em risco (Thrusfield 2004). Há um número razoável de estudos demográficos que caracterizaram populações caninas. Estudos descritivos sobre as raças, o gênero e as idades dos cães já foram realizados em países das Américas (Fielding \& Plumridge 2005, Fiorello et al. 2006, Acosta-Jamett et al. 2010, Pulczer et al. 2013), Europa (Slater et al. 2008, Downes et al. 2009, Murray et al. 2010), África (Butler \& Bingham 2000, Kitala et al. 2001, Ratsitorahina et al. 2009) e Ásia (Matter et al. 2000, Pal 2001, Kongkaew et al. 2004) e visam, principalmente, o estabelecimento de programas de controle de doenças. No entanto, não existem dados publicados acerca das características da população canina que habita o estado do Rio Grande do Sul (RS). Também são escassos, os estudos que analisam as populações de quaisquer outras regiões do Brasil (Nunes et al. 1997, Dias et al. 2004, Canatto et al. 2012).

Conhecer a população de cães encaminhada para necropsia em um serviço de diagnóstico anatomopatológico post mortem é uma ferramenta no estudo de diferentes aspectos das doenças locais, e assemelha-se, em alguns aspectos, aos estudos descritivos populacionais citados no parágrafo anterior. Embora a população de necropsias não seja um reflexo fidedigno da população que habita a região, conhecer as suas características é importante no estudo epidemiológico de doenças diagnosticadas localmente, particularmente daquelas doenças que causam a morte ou justificam a eutanásia. Para se afirmar que os cães de uma determinada raça morrem mais frequentemente por câncer de pele do que os cães de outras raças, por exemplo, deve-se conhecer, entre outros dados, o número total de cães de cada raça necropsiados no período estudado. Diante da necessidade em se estabelecer um perfil da população canina recebida para realização de necropsias no Laboratório de Patologia Veterinária (LPV-UFSM) da Universidade Federal de Santa Maria (UFSM), o objetivo deste estudo é caracterizar essa população canina quanto ao gênero, à raça e à idade, com a finalidade de disponibilizar um banco de dados a partir dos cães necropsiados, já que dificilmente teremos, pelo menos em curto prazo, como estabelecer os mesmos achados da população total de cães da Região Central do RS. Os resultados aqui apresentados servirão como um subsídio comparativo para futuros estudos sobre epidemiologia das doenças de cães da Região Central do RS, auxiliando para uma mais correta compreensão e interpretação dos resultados encontrados. 


\section{MATERIAL E MÉTODOS}

Os laudos de exame de necropsia de cães, realizadas entre 1964 e 2013 no LPV-UFSM, foram revisados, e deles foram retiradas informações referentes ao gênero, à idade e às raças de todos os cães oriundos dos municípios que compõem a Região Central do RS. Quanto ao gênero, os cães foram classificados apenas como macho e fêmea, independentemente de serem castrados ou não. A idade foi contabilizada quanto ao número de anos. Idades expressas em meses (ex.: 24 meses) foram convertidas em anos (no caso do exemplo anterior, dois anos). Em cães com idades expressas em anos e meses (ex.: um ano e três meses), o número de meses foi desconsiderado. Quanto às raças, os cães foram classificados em sem raça definida (SRD) ou de raça definida (RD). Cães mestiços, do cruzamento de duas RD, foram incluídos como SRD. As raças de cães necropsiados foram consideradas apenas a partir de 1974, visto que antes deste ano, dados referentes à raça não constavam nos laudos de necropsia. Somente foram incluídas raças homologadas pela Fédération Cynologique Internationale (FCI 2015) e/ou pelo American Kennel Club (AKC 2015). As raças não reconhecidas por nenhuma dessas associações (FCI e AKC), mas que fazem parte do Grupo 11 ("raças não reconhecidas pela FCI") da Confederação Brasileira de Cinofilia (CBKC 2015) também foram contempladas. Para fins de comparação, este estudo foi dividido em cinco décadas: 1964-1973, 1974-1983, 1984-1993, 1994-2003 e 2004-2013.

As análises estatísticas descritivas foram realizadas através do programa Microsoft ${ }^{\circledR}$ Office Excel $^{\circledR} 2010$ e SPSS.

\section{RESULTADOS}

No período que compreende os anos entre 1964 e 2013, foram localizados 7.780 laudos de exame de necropsia de cães. Uma comparação gráfica da percentagem de necropsias por década pode ser contemplada na Figura 1. Dos cães cujo gênero foi informado nos laudos (n=7.634), $4.012(52,6 \%)$ eram machos e $3.622(47,4 \%)$ eram fêmeas. A proporção de machos e fêmeas na população ao longo das cinco décadas pode ser observada na Figura 2.

A idade dos cães variou de um dia a 22 anos de vida (mediana $=3$ anos). A Figura 3 demonstra a distribuição do número de cães necropsiados pelas idades de morte. Com a Figura 4 é possível visualizar essa distribuição ao longo das décadas. Dos cães cuja raça foi informada nos laudos ( $\mathrm{n}=6.885)$, 4.119 (59,8\%) eram de RD e 2.766 (40,2\%) não tinham raça definida. A proporção de cães de RD e SRD na população ao longo das quatro décadas foi de: 56,7\% (RD)/43,3\% (SRD) (1974-1983), 54,1\% (RD)/45,9\% (SRD) (1984-1993), 60,5\%(RD)/39,5\% (SRD) (1994-2003) e 62,6\%(RD)/37,4\% (SRD) (2004-2013). Foram observados cães de 66 raças diferentes, das quais 63 são reconhecidas pela FCI e 61 pelo AKC. Das 66 raças observadas, as mais frequentes foram: Pastor Alemão ( $n=710[17,2 \%])$, Poodle ( $n=365[8,9 \%])$, Boxer ( $n=285$ [6,9\%]), Dachshund ( $n=259$ [6,3\%]), Pinscher Miniatura $(n=230[5,6 \%])$, Rottweiler $(n=220[5,3 \%])$, Fila Brasileiro $(n=189$ [4,6\%]), Cocker Spaniel Inglês $(n=185[4,5 \%])$, Pointer Inglês $(n=162[3,9 \%])$, Collie Pelo Longo $(n=154[3,7 \%])$, Pequinês $(n=138[3,4 \%])$ e Yorkshire Terrier ( $n=135$ [3,3\%]), Terrier Brasileiro ( $n=117$ [2,8\%]), e Shih Tzu (n=61 [1,5\%]). A frequência de ocorrência das principais raças de cães necropsiados ao longo das quatro décadas pode ser observada na Figura 5 (raças de cães de porte grande e gigante) e na Figura 6 (raças de cães de porte

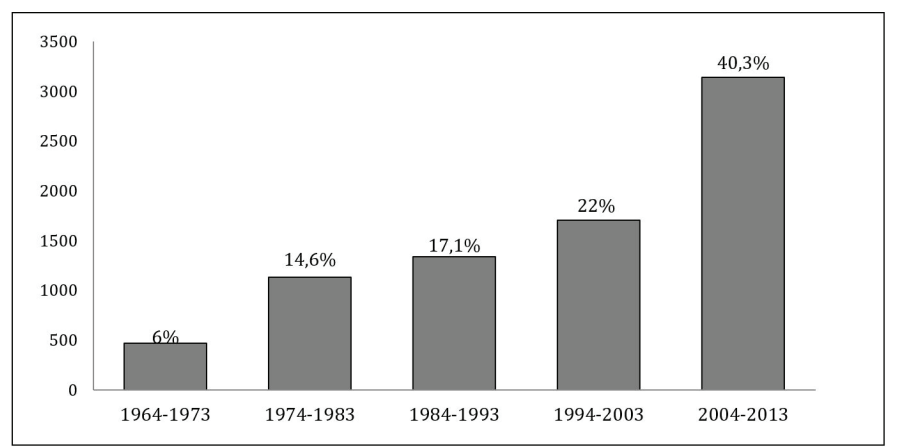

Fig.1. Percentagem de cães necropsiados por década no Laboratório de Patologia Veterinária, Universidade Federal de Santa Maria (1964-2013).

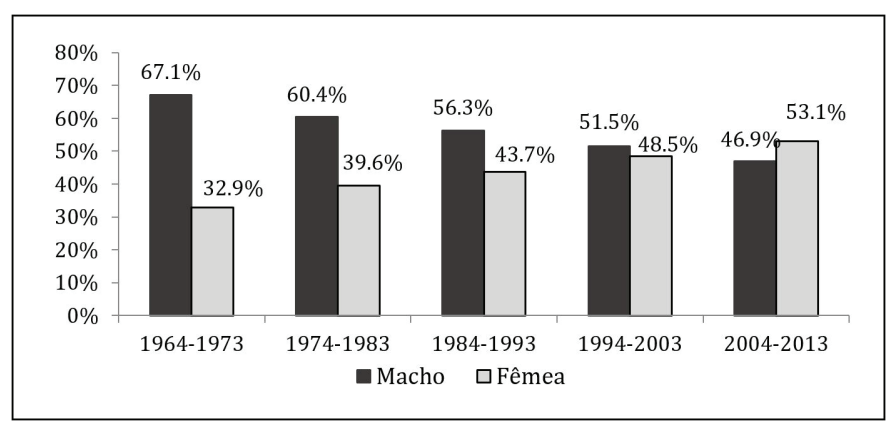

Fig.2. Gênero dos cães necropsiados por década no Laboratório de Patologia Veterinária, Universidade Federal de Santa Maria (1964-2013).

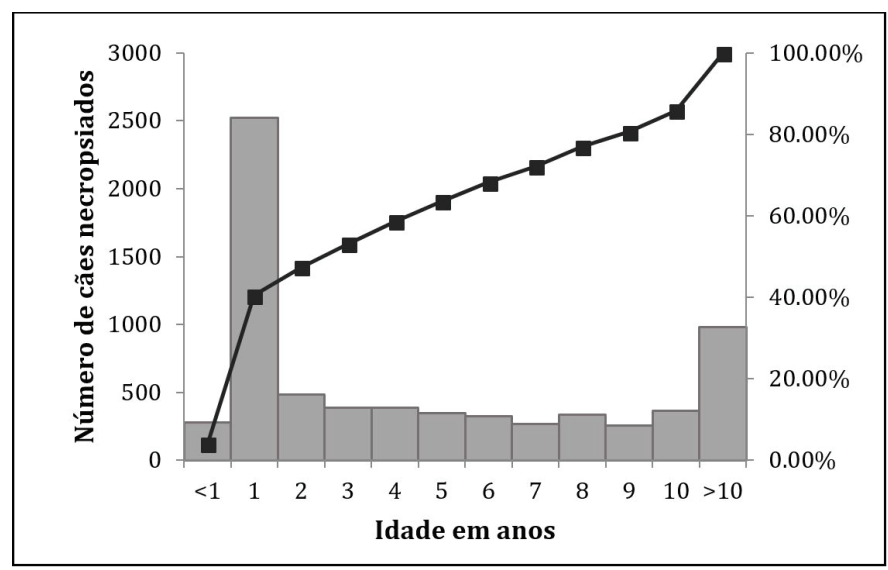

Fig.3. Distribuição do número total de cães necropsiados no Laboratório de Patologia Veterinária, Universidade Federal de Santa Maria (1974-2013), de acordo com a idade de morte.

pequeno e médio). As principais mudanças na ocorrência das raças ao longo das décadas deste estudo foram, dentre as mais marcantes nas raças de porte grande e gigante: a diminuição na percentagem de Pastor Alemão, Pointer Inglês e Collie Pelo Longo e o aumento na proporção de Rottweiler, Boxer e Labrador Retriever. E dentre as raças de porte pequeno e médio, o aumento na frequência de Poodle, Dachshund, 


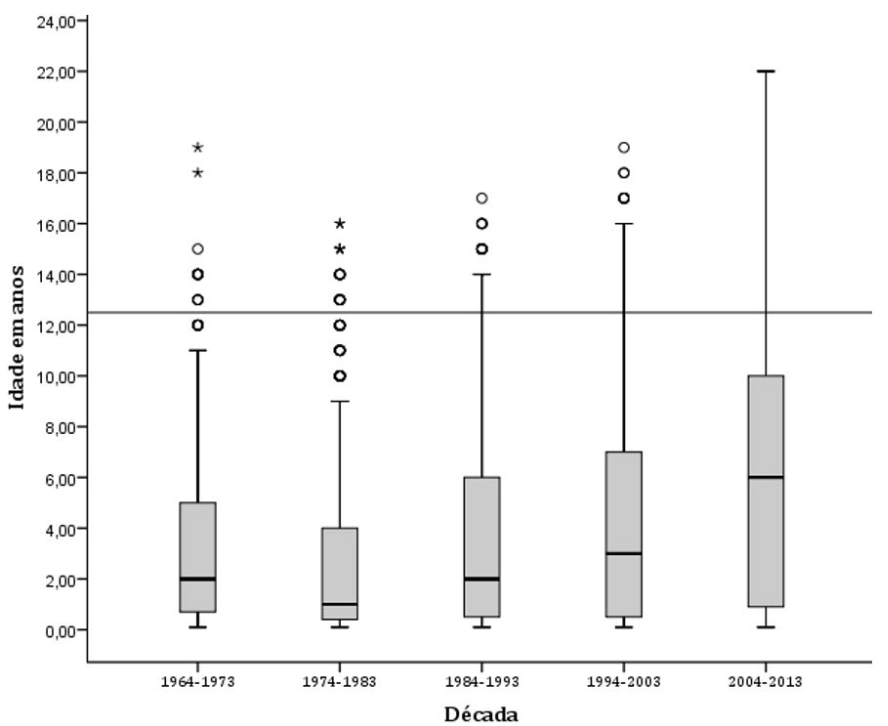

Fig.4. Distribuição do número de cães necropsiados por década no Laboratório de Patologia Veterinária, Universidade Federal de Santa Maria (1974-2013), de acordo com a idade de morte.

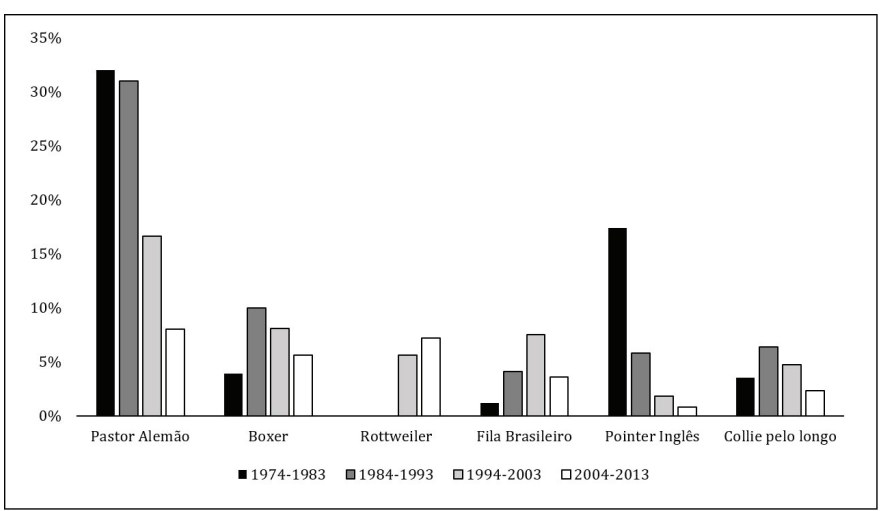

Fig.5. Percentagem das principais raças de cães de porte grande e gigante necropsiados por década no Laboratório de Patologia Veterinária, Universidade Federal de Santa Maria (1974-2013).

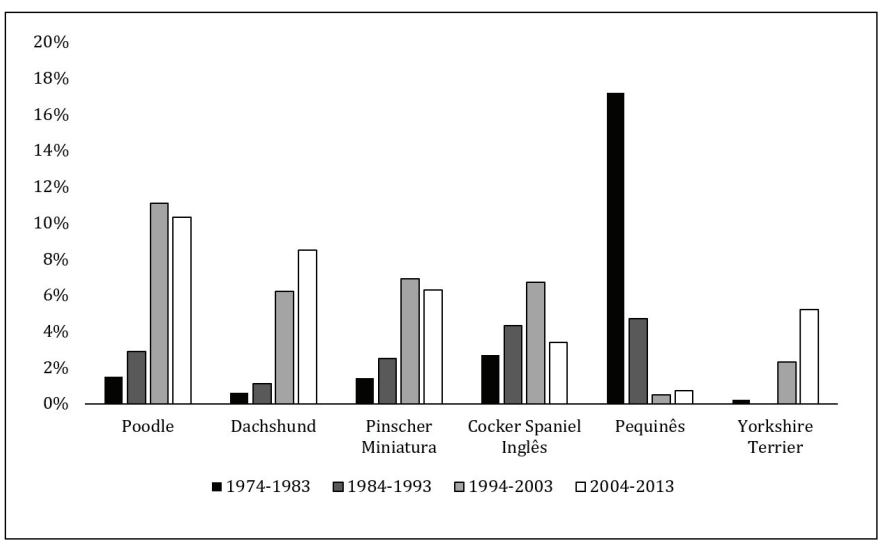

Fig.6. Percentagem das principais raças de cães de portes pequeno e médio necropsiados por década no Laboratório de Patologia Veterinária, Universidade Federal de Santa Maria (1974-2013).
Pinscher Miniatura e Yorkshire Terrier e uma diminuição na ocorrência de Terrier Brasileiro e Pequinês. 0 ranking das raças mais frequentemente necropsiadas por década no LPV-UFSM pode ser visualizado no Quadro 1.

\section{DISCUSSÃO}

Através dos resultados, puderam-se observar mudanças no perfil da população de cães necropsiados no LPV-UFSM ao longo dos últimos 50 anos. Determinar as características populacionais de cães que habitam a Região Central do RS está muito além do alcance desse estudo, entretanto, ao longo da discussão, iremos comparar alguns resultados observados a partir dos cães necropsiados no LPV-UFSM com perfis de populações vivas e necropsiadas em outras partes do mundo e discutir acerca das diferenças e semelhanças entre estas populações.

Ao longo dos anos, observou-se um crescimento gradual da frequência na população de cães necropsiados no LPV-UFSM. Dos 7.780 cães incluídos neste estudo, 6\% foram necropsiados na primeira década (1964-1973), enquanto cerca de $40 \%$ foram necropsiados na última (2004-2013). As hipóteses para o aumento da população necropsiada devem incluir: 1) uma maior divulgação e reconhecimento dos serviços prestados ao longo dos anos; 2) o crescimento do número de cães atendidos em consultórios e clínicas particulares e no Hospital Veterinário Universitário (HVU) da UFSM, o que leva, consequentemente, a uma maior busca por diagnósticos post mortem; e/ou 3) o crescimento da população de cães que habita nossa região nos últimos anos. Infelizmente não há como comprovar ou refutar nenhuma dessas hipóteses.

Uma das principais transformações observadas no perfil epidemiológico da população de cães necropsiados no LPV-UFSM ao longo do tempo foi o crescimento do número de fêmeas, gerando uma proporção muito semelhante entre machos e fêmeas na última década (2004-2013). Enquanto as cadelas correspondiam a apenas cerca de 33\% da população do laboratório na primeira década (1964-1973), elas passaram a representar aproximadamente $53 \%$ dessa mesma população nos últimos dez anos (2004-2013). Alguns estudos realizados em populações de cães vivos da Suécia (Egenvall et al. 2000), do Zimbábue (Butler \& Bingham 2000), das Bahamas (Fielding \& Plumridge 2005), da Bolívia (Fiorello et al. 2006) e da Irlanda (Downes et al. 2009) não detectaram diferenças significativas entre o número de machos e fêmeas dos grupos analisados. Já estudos conduzidos no Brasil (Nunes et al. 1997, Canatto et al. 2012), na Índia (Pal 2001), no Quênia (Kitala et al. 2001), na Itália (Slater et al. 2008), em Madagascar (Ratsitorahina et al. 2009) e na Inglaterra (O'Neill et al. 2013) relataram uma discreta predominância de machos. Ainda outros levantamentos populacionais, realizados no Sri Lanka (Matter et al. 2000), na Tailândia (Kongkaew et al. 2004), no Chile (Acosta-Jamett et al. 2010) e na Guatemala (Pulczer et al. 2013), demonstraram uma grande proporção de machos nas populações analisadas. Daniels (1983) chegou a relatar uma proporção de três machos para cada fêmea em um antigo estudo realizado nos Estados Unidos. Mesmo com a predominância de machos nos estudos anteriormente citados, não é possível inferir que a proporção de machos está aumentando ou diminuindo nestes grupos ao longo do tempo, pois todos estes estudos são estáticos, tornando difícil confrontar os resultados encontrados por esses autores 
Quadro 1. Ranking das principais raças necropsiadas por década no Laboratório de Patologia Veterinária, Universidade Federal de Santa Maria (1964-2013)

\begin{tabular}{|c|c|c|c|c|c|c|c|c|}
\hline Posição & 1974-1983 & $\mathrm{N}(\%)$ & 1984-1993 & $\mathrm{N}(\%)$ & $1994-2003$ & $\mathrm{~N}(\%)$ & 2004-2013 & $\mathrm{N}(\%)$ \\
\hline & Total RD & $517(100 \%)$ & Total RD & $653(100 \%)$ & Total RD & $1.006(100 \%)$ & Total RD & $1.943(100 \%)$ \\
\hline $1^{\mathrm{a}}$ & Pastor Alemão & $165(32 \%)$ & Pastor Alemão & 203 (31\%) & Pastor Alemão & $167(16,6 \%)$ & Poodle & $201(10,3 \%)$ \\
\hline $2^{\underline{a}}$ & Pointer Inglês & $90(17,4 \%)$ & Dobermann & $66(10 \%)$ & Poodle & $112(11,1 \%)$ & Dachshund & $166(8,5 \%)$ \\
\hline $3^{\mathrm{a}}$ & Pequinês & $89(17,2 \%)$ & Boxer & $62(10 \%)$ & Boxer & $81(8,1 \%)$ & Pastor Alemão & $156(8 \%)$ \\
\hline $4^{\mathrm{a}}$ & $\begin{array}{c}\text { Terrier } \\
\text { Brasileiro }\end{array}$ & $41(7,9 \%)$ & $\begin{array}{l}\text { Collie Pelo } \\
\text { Longo }\end{array}$ & $42(6,4 \%)$ & Fila Brasileiro & $76(7,6 \%)$ & Rottweiler & $139(7,2 \%)$ \\
\hline $5^{\mathrm{a}}$ & Boxer & $20(3,9 \%)$ & Pointer Inglês & $38(5,8 \%)$ & $\begin{array}{l}\text { Pinscher } \\
\text { Miniatura }\end{array}$ & $69(6,9 \%)$ & $\begin{array}{l}\text { Pinscher } \\
\text { Miniatura }\end{array}$ & $122(6,3 \%)$ \\
\hline $6^{\underline{a}}$ & $\begin{array}{l}\text { Collie Pelo } \\
\text { Longo }\end{array}$ & $18(3,5 \%)$ & Pequinês & $31(4,7 \%)$ & $\begin{array}{l}\text { Cocker Spaniel } \\
\text { Inglês }\end{array}$ & $67(6,7 \%)$ & Boxer & $109(5,6 \%)$ \\
\hline $7 \underline{a}$ & Dobermann & $17(3,3 \%)$ & $\begin{array}{c}\text { Terrier } \\
\text { Brasileiro }\end{array}$ & $30(4,6 \%)$ & Dachshund & $62(6,2 \%)$ & $\begin{array}{l}\text { Yorkshire } \\
\text { Terrier }\end{array}$ & $101(5,2 \%)$ \\
\hline $8^{\underline{a}}$ & $\begin{array}{c}\text { Cocker } \\
\text { Spaniel Inglês }\end{array}$ & $14(2,7 \%)$ & $\begin{array}{l}\text { Cocker Spaniel } \\
\text { Inglês }\end{array}$ & $28(4,3 \%)$ & Rottweiler & $56(5,6 \%)$ & $\begin{array}{l}\text { Labrador } \\
\text { Retriever }\end{array}$ & $71(3,7 \%)$ \\
\hline $9 \underline{a}$ & Dálmata & $11(2,1 \%)$ & Fila Brasileiro & $27(4,1 \%)$ & Collie Pelo Longo & $47(4,7 \%)$ & Fila Brasileiro & $70(3,6 \%)$ \\
\hline $10^{\mathrm{a}}$ & Poodle & $8(1,5 \%)$ & Poodle & $19(2,9 \%)$ & Dobermann & $41(4,1 \%)$ & $\begin{array}{l}\text { Cocker Spaniel } \\
\text { Inglês }\end{array}$ & $66(3,4 \%)$ \\
\hline $11^{\mathrm{a}}$ & $\begin{array}{l}\text { Pinscher } \\
\text { Miniatura }\end{array}$ & $7(1,4 \%)$ & $\begin{array}{l}\text { Husky } \\
\text { Siberiano }\end{array}$ & $18(2,8 \%)$ & Terrier Brasileiro & $23(2,3 \%)$ & Shih Tzu & $58(3 \%)$ \\
\hline $12^{\mathrm{a}}$ & Fila Brasileiro & $6(1,2 \%)$ & $\begin{array}{l}\text { Pinscher } \\
\text { Miniatura }\end{array}$ & $16(2,5 \%)$ & Yorkshire Terrier & $23(2,3 \%)$ & $\begin{array}{l}\text { Collie Pelo } \\
\text { Longo }\end{array}$ & $44(2,3 \%)$ \\
\hline $13^{\underline{a}}$ & Dogue Alemão & $5(1 \%)$ & Dogue Alemão & $12(1,8 \%)$ & Dogue Alemão & $20(2 \%)$ & Maltês & $29(1,5 \%)$ \\
\hline $14^{\underline{a}}$ & Weimaraner & $5(1 \%)$ & Dálmata & $9(1,4 \%)$ & Dálmata & $18(1,8 \%)$ & Dálmata & $27(1,4 \%)$ \\
\hline $15^{\underline{a}}$ & Setter Inglês & $4(0,8 \%)$ & Basset Hound & $7(1,1 \%)$ & Pointer Inglês & $18(1,8 \%)$ & Dobermann & $27(1,4 \%)$ \\
\hline $16^{\mathrm{a}}$ & Afghan Hound & $3(0,6 \%)$ & Dachshund & $7(1,1 \%)$ & Husky Siberiano & $14(1,4 \%)$ & Chow Chow & $26(1,3 \%)$ \\
\hline $17 \underline{a}$ & Dachshund & $3(0,6 \%)$ & Pastor Belga & $6(0,9 \%)$ & Pastor Belga & $11(1,1 \%)$ & $\begin{array}{c}\text { American Pit } \\
\text { Bull Terrier }\end{array}$ & $25(1,3 \%)$ \\
\hline $18^{\mathrm{a}}$ & Basset Hound & $2(0,4 \%)$ & Setter Inglês & $5(0,8 \%)$ & Akita & $9(0,9 \%)$ & Border Collie & $22(1,1 \%)$ \\
\hline $19 \underline{a}$ & Bulldog & $2(0,4 \%)$ & Rottweiler & $4(0,6 \%)$ & $\begin{array}{l}\text { Old English } \\
\text { Sheepdog }\end{array}$ & $9(0,9 \%)$ & $\begin{array}{c}\text { Terrier } \\
\text { Brasileiro }\end{array}$ & $19(1 \%)$ \\
\hline $20^{\mathrm{a}}$ & Beagle & $1(0,2 \%)$ & Akita & $3(0,5 \%)$ & $\begin{array}{l}\text { Labrador } \\
\text { Retriever }\end{array}$ & $8(0,8 \%)$ & Beagle & $17(0,9 \%)$ \\
\hline $21^{\mathrm{a}}$ & Chihuahua & $1(0,2 \%)$ & Galgo Espanhol & $3(0,5 \%)$ & São Bernardo & $7(0,7 \%)$ & São Bernardo & $17(0,9 \%)$ \\
\hline $22^{\underline{a}}$ & $\begin{array}{l}\text { Husky } \\
\text { Siberiano }\end{array}$ & $1(0,2 \%)$ & São Bernardo & $3(0,5 \%)$ & Beagle & $6(0,6 \%)$ & Pointer Inglês & $16(0,8 \%)$ \\
\hline $23^{\mathrm{a}}$ & São Bernardo & $1(0,2 \%)$ & Weimaraner & $3(0,5 \%)$ & Weimaraner & $6(0,6 \%)$ & Bulldog & $15(0,8 \%)$ \\
\hline $24^{\underline{a}}$ & Schnauzer & $1(0,2 \%)$ & Afghan Hound & $2(0,3 \%)$ & Basset Hound & $5(0,5 \%)$ & Dogue Alemão & $15(0,8 \%)$ \\
\hline $25^{\underline{a}}$ & Whippet & $1(0,2 \%)$ & Beagle & $2(0,3 \%)$ & Chihuahua & $5(0,5 \%)$ & Husky Siberiano & $13(0,7 \%)$ \\
\hline $26^{\mathrm{a}}$ & $\begin{array}{c}\text { Yorkshire } \\
\text { Terrier }\end{array}$ & $1(0,2 \%)$ & Chihuahua & $2(0,3 \%)$ & Chow Chow & $5(0,5 \%)$ & Lhasa Apso & $13(0,7 \%)$ \\
\hline $27^{a}$ & - & - & $\begin{array}{l}\text { Old English } \\
\text { Sheepdog }\end{array}$ & $2(0,3 \%)$ & Shar Pei & $5(0,5 \%)$ & Pequinês & $13(0,7 \%)$ \\
\hline $28^{\mathrm{a}}$ & - & - & Bull Terrier & $1(0,2 \%)$ & Bulldog & $4(0,4 \%)$ & Shar Pei & $12(0,6 \%)$ \\
\hline $29 \underline{a}$ & - & - & Chow Chow & $1(0,2 \%)$ & Lhasa Apso & $4(0,4 \%)$ & Akita & $11(0,6 \%)$ \\
\hline $30^{\mathrm{a}}$ & - & - & Greyhound & $1(0,2 \%)$ & Pequinês & $4(0,4 \%)$ & Chihuahua & $10(0,5 \%)$ \\
\hline
\end{tabular}

com aqueles aqui expressos. De qualquer forma, podemos perceber que a predominância de machos em populações vivas de cães é ainda muito descrita na literatura, e atribuída por muitos autores: 1) à preferência do público por cães desse gênero (Daniels 1983, Kitala et al. 2001, Hsu et al. 2003, Ratsitorahina et al. 2009, Acosta-Jamett et al. 2010); 2) à maior mortalidade de fêmeas (Cleaveland 1996, Butler \& Bingham 2000, Kitala et al. 2001, Pulczer et al. 2013) e/ou; $3)$ à maior natalidade de machos ( $\mathrm{Pal}$ 2001), citada por um autor como variando de $51 \%$ a 56\% (Mialot 1988).
A mediana de idade dos cães necropsiados no LPV-UFSM foi de 3 anos. Neste estudo, foi utilizado o parâmetro "mediana" para a análise devido à característica da distribuição da variável "idade" ao longo das décadas. Na literatura mundial, já foram relatadas "idades medianas de morte" de 11 e 12 anos no Reino Unido (Michell 1999, Adams et al. 2010, O'Neill et al. 2013), 10 anos na Dinamarca (Proschowsky et al. 2003), 8,5 anos nos Estados Unidos (Patronek et al. 1997,), 3 anos no Brasil (Bentubo et al. 2007). As distorções observadas entre as medianas de idade de diferentes estudos podem 
ser atribuídas a diferenças na metodologia adotada por cada autor e a variações nas características das populações analisadas. Grupos de cães atendidos em clínicas e hospitais (Lund et al. 1999) ou com morte súbita (Olsen \& Allen 2000), por exemplo, geralmente têm uma idade menor de morte quando comparados a grupos selecionados aleatoriamente (Michell 1999).

Algumas características das populações analisadas também podem ter influência na mediana de idade de morte. A massa corporal dos cães, por exemplo, tem uma relação inversa com a expectativa de vida (Greer et al. 2007), o que significa dizer que os cães de raças de porte pequeno têm, geralmente, uma maior expectativa de vida quando comparados aos de porte grande (Patronek et al. 1997, Michell 1999, Egenvall et al. 2000, Proschowsky et al. 2003, Greer et al. 2007, Adams et al. 2010, Fleming et al. 2011, O’Neill et al. 2013). Além do peso corporal, há fatores genéticos que aumentam ou diminuem a expectativa de vida para diferentes raças, como é o exemplo dos cães Boxer, que têm uma grande probabilidade de morrerem antes dos 10 anos de idade, ou seja, uma expectativa de vida menor quando comparada a cães de outras raças, mas de mesmo porte (Egenvall et al. 2000). 0 terceiro fator que pode causar distorções na mediana de idade de morte de uma população é o país onde o estudo foi realizado. Em países em desenvolvimento, a proporção de cães vacinados e castrados é pequena, os cães visitam menos o veterinário, e frequentemente tem acesso irrestrito a rua (Butler \& Bingham 2000, Matter et al. 2000, Ratsitorahina et al. 2009, Acosta-Jamett et al. 2010, Canatto et al. 2012), fatores que podem contribuir com mortes relacionadas a agentes infecciosos e traumatismos (Bentubo et al. 2007, Acosta-Jamett et al. 2010). Por esses motivos, a morte por agentes infecciosos é altamente prevalente no Brasil, o que já foi demonstrado em São Paulo (Bentubo et al. 2007) e no RS (Fighera et al. 2008), e pode diminuir significativamente a expectativa de vida das populações de cães que habitam esses países, influenciando a mediana de idade de morte dos cães que chegam para necropsia.

0 crescimento na mediana ao longo das cinco décadas pode indicar que a expectativa de vida da população de cães que habita a Região Central do RS esteja aumentando. A falta de dados referentes à população total de cães que habitam essa região impede que se confirme ou refute tal teoria. No entanto, alguns autores afirmam que os cães realmente têm vivido mais nos dias de hoje (Withrow et al. 2013). É muito provável que o aumento da expectativa de vida refletirá, a médio e longo prazo, no aumento da incidência de determinadas doenças relacionadas à senilidade, como por exemplo, o câncer e as doenças degenerativas. 0 aumento da incidência do câncer em humanos tem uma relação muito bem estabelecida com o crescente aumento na expectativa de vida dos diferentes países (Mukherjee 2012) e isso tem sido frequentemente extrapolado para cães (Withrow et al. 2013). Outro ponto importante na análise da idade foi o pico de ocorrência de cães com 1 e 10 anos de idade (Figura 3). Os motivos para este aumento pontual na frequência destas duas idades permanecem desconhecidos.

Discorrer detalhadamente acerca da representatividade das raças na população de cães necropsiados no LPV-UFSM e dos motivos da preferência por determinadas raças vai muito além dos propósitos deste estudo. No entanto, discutiremos aqui alguns pontos que consideramos importantes em relação às mudanças observadas ao longo dos anos. Um levantamento do AKC sobre as raças de cães mais comuns nos Estados Unidos em 2013 incluiu, em ordem decrescente de frequência, Labrador Retriever, Pastor Alemão, Golden Retriever, Boxer, Poodle, Rottweiler e Dachshund na lista das 10 raças mais populares entre os norte-americanos. Interessantemente, todas essas raças, com exceção do Dachshund, também apareceram entre as mais comuns em um estudo de mortalidade baseado em informações de diversas clínicas norte-americanas (Fleming et al. 2011), sugerindo que a população que morreu e foi incluída no estudo de Fleming et al. (2011) tenha sido bastante semelhante à população total de cães que habita o país. Seis das raças citadas na lista do AKC e cinco das mais comuns no estudo de Fleming et al. (2011) também estão entre as primeiras raças mais comumente necropsiadas no LPV-UFSM na última década e demonstradas no Quadro 1, ou seja: Pastor Alemão, Poodle, Boxer, Dachshund, Rottweiler e Labrador Retriever. Muitas dessas raças também aparecem com frequência liderando as listas de raças mais comuns em outros estudos de mortalidade realizados na Inglaterra (Michell 1999), Suécia (Egenvall et al. 2000, Bonnett et al. 2005) e Dinamarca (incluindo aqui o Cocker Spaniel Inglês) (Proschowsky et al. 2003) e em estudos de popularidade, como o realizado pelo Canadian Kennel Club (CKC 2007).

Apesar das mudanças na frequência de ocorrência de diferentes raças no LPV-UFSM ao longo das décadas, observou-se apenas um discreto aumento na proporção de cães de RD no LPV-UFSM nos últimos 40 anos. Na segunda década (1974-1983), havia 56,7\% de cães de RD na população, enquanto na última (2004-2013), a percentagem foi de 62,6\%, uma variação muito pequena. No trabalho de Slater et al. (2008), cães de RD correspondiam a $40 \%$ da população da Itália. A maioria dos estudos populacionais, no entanto, não inclui a raça em sua coleta de dados. Estudos de mortalidade semelhantes a este, realizados na Inglaterra (O'Neill et al. 2013) e nos Estados Unidos (Fleming et al. 2011), relatam que $77,9 \%$ e 75,4\% dos cães incluídos, respectivamente, eram de RD; resultados até certo ponto próximos à percentagem aqui descrita para os últimos 10 anos (2004-2013).

As informações obtidas neste estudo retrospectivo devem ser interpretadas com cautela, principalmente porque o grupo de cães que chega para realização de necropsia no LPV-UFSM é oriundo, predominantemente, de clínicas, consultórios e hospitais veterinários. Além de que todos os cães incluídos morreram ou foram eutanasiados em decorrência de diferentes doenças. Isto significa dizer que a população aqui analisada não é um reflexo fidedigno da população total que habita a Região Central do RS, mas, possivelmente, aproxime-se dessa.

\section{CONCLUSÕES}

Os dados aqui apresentados demonstram que a população de cães necropsiados sofreu mudanças importantes no que diz respeito ao gênero, à idade e às raças mais comuns em cada década.

O conhecimento destas alterações é fundamental no estudo epidemiológico dos distúrbios que causam a morte ou justificam a eutanásia de cães.

Algumas mudanças ocorridas na estrutura da população de necropsias, como por exemplo, o aumento na proporção de idosos, irão influenciar diretamente na incidência das diferentes doenças diagnosticadas no LPV-UFSM. 
Espera-se que este estudo traga resultados úteis para comparação com muitos outros acerca de doenças de cães, tanto no Rio Grande do Sul quanto no restante do Brasil.

A realização de estudos semelhantes a este em populações de cães necropsiados ao longo de um extenso período em outras regiões e países ajudará a reforçar, confirmar ou refutar algumas das hipóteses aqui propostas no que diz respeito às mudanças relacionadas ao gênero, à idade e à raça dos cães pertencentes à população da Região Central do Rio Grande do Sul.

Agradecimentos.- Ao professor Luís Gustavo Corbellini, Universidade Federal do Rio Grande do Sul (UFRGS), pelas sugestões, principalmente acerca dos diferentes vieses deste estudo. Mariana Martins Flores é bolsista da Coordenação de Aperfeiçoamento de Pessoal de Nível Superior (CAPES).

\section{REFERÊNCIAS}

Acosta-Jamett G., Cleaveland S., Cunningham A.A. \& Bronsvoort B.M.D. 2010. Demography of domestic dogs in rural and urban areas of the Coquimbo region of Chile and implications for disease transmission. Prev. Vet. Med. 94(3/4):272-281. http://dx.doi.org/10.1016/j.prevetmed.2010.01.002. PMid:20096943.

Adams V.J., Evans K.M., Sampson J. \& Wood J.L. 2010. Methods and mortality results of a health survey of purebred dogs in the UK. J. Small Anim. Pract. 51(10):512-524. http://dx.doi.org/10.1111/j.1748-5827.2010.00974.x. PMid:21029096.

AKC 2015. Dog Owners, Breeds. American Kennel Club. Disponível em <http:// www.akc.org/breeds/index.cfm> Acesso em 5 jan. 2015.

Bentubo H.D.L., Tomaz M.A., Bondan E.F. \& Lallo M.A. 2007. Expectativa de vida e causas de morte em cães da área metropolitana de São Paulo, Brasil. Ciência Rural 37(4):1021-1026. http://dx.doi.org/10.1590/S010384782007000400016.

Bonnett B.N., Egenvall A., Hedhammar A. \& Olson P. 2005. Mortality in over 350,000 insured Swedish dogs from 1995-2000: I. Breed-, gender-, ageand cause-specific rates. Acta Vet. Scand. 46(3):105-120. http://dx.doi. org/10.1186/1751-0147-46-105. PMid:16261924.

Butler J.R.A. \& Bingham J. 2000. Demography and dog-human relationships of the dog population in Zimbabwean communal lands. Vet. Rec. 147(16):442446. http://dx.doi.org/10.1136/vr.147.16.442. PMid:11079440.

Canatto B.D., Silva E.A., Bernardi F., Mendes M.C.N.C., Paranhos N.T. \& Dias R.A. 2012. Caracterização demográfica das populações de cães e gatos supervisionados do município de São Paulo. Arq. Bras. Med. Vet. Zootec. 64(6):1515-1523. http://dx.doi.org/10.1590/S0102-09352012000600017.

CBKC 2015. Raças Não Reconhecidas Pela FCI. Confederação Brasileira de Cinofilia. Disponível em <http://www.cbkc.org/padroes/grupo11.html> Acesso em 5 jan. 2015.

CKC 2007. Top 20 CKC Breeds. Canadian Kennel Club. Disponível em: <http:// web.archive.org/web/20081230161349/http://www.ckc.ca/en/portals/0/ pdf/Top\%2020\%20Breeds_2007.pdf> Acesso em 13 jan. 2015.

Cleaveland S. 1996. The epidemiology of rabies and canine distemper in the Serengeti, Tanzania. PhD Thesis, Department of Medical Parasitology, London School of Hygiene and Tropical Medicine, University of London, London. 267p.

Daniels T.J. 1983. The social organization of free-ranging urban dogs. I. Nonestrous social behavior. Appl. Anim. Ethol. 10(4):341-363. http://dx.doi. org/10.1016/0304-3762(83)90184-0.

Dias R.A., Garcia R.C., Silva D.F., Amaku M., Ferreira Neto J.S. \& Ferreira F. 2004. Estimativa de populações canina e felina domiciliadas em zona urbana do Estado de São Paulo. Revta Saúde Pública 38(4):565-570. http://dx.doi. org/10.1590/S0034-89102004000400013.

Downes M., Canty M.J. \& More S.J. 2009. Demography of the pet dog and cat population on the island of Ireland and human factors influencing pet ownership. Prev. Vet. Med. 92(1/2):140-149. http://dx.doi.org/10.1016/j. prevetmed.2009.07.005. PMid:19700212.

Egenvall A., Hedhammar A., Bonnett B.N. \& Olson P. 2000. Gender, age, breed and distribution of morbity and morbality in insured dogs in Sweden during 1995 and 1996. Vet. Rec. 146(18):519-525. http://dx.doi.org/10.1136/ vr.146.18.519. PMid:11321213.

FCI 2015. FCI Breeds Nomenclature. Fédération Cynologique Internationale. Disponível em <http://www.fci.be/en/Nomenclature/> Acesso em 5 jan. 2015.

Fielding W.J. \& Plumridge S.J. 2005. Characteristics of owned dogs on the island of New Providence, The Bahamas. J. Appl. Anim. Welf. Sci. 8(4):245260. http://dx.doi.org/10.1207/s15327604jaws0804_2.PMid:16436029.

Fighera R.A., Souza T.M., Silva M.C., Brum J.S., Graça D.L., Kommers G.D., Irigoyen L.F. \& Barros C.S.L. 2008. Causas de morte e razões para eutanásia de cães da Mesorregião do Centro Ocidental Rio-Grandense (1965-2004). Pesq. Vet. Bras. 28(4):223-230. http://dx.doi.org/10.1590/S0100736X2008000400005.

Fiorello C., Noss A.J. \& Deem S.L. 2006. Demography, hunting ecology, and pathogen exposure of domestic dogs in the isoso of Bolivia. Conserv. Biol. 20(3):762-771. http://dx.doi.org/10.1111/j.1523-1739.2006.00466.x. PMid:16909569.

Fleming J.M., Creevy K.E. \& Promislow D.E.L. 2011. Mortality in North American dogs from 1984 to 2004: an investigation into age-, size- and breed-related causes of death. J. Vet. Intern. Med. 25(2):187-198. http:// dx.doi.org/10.1111/j.1939-1676.2011.0695.x. PMid:21352376.

Greer K.A., Canterberry S.C. \& Murphy K.E. 2007. Statistical analysis regarding the effects of height and weight on life span of the domestic dog. Res. Vet. Sci. 82(2):208-214. http://dx.doi.org/10.1016/j.rvsc.2006.06.005. PMid:16919689.

Hsu Y., Liu Severinghaus L. \& Serpell J.A. 2003. Dog keeping in Taiwan: its contribution to the problem of free-roaming dogs. J. Appl. Anim. Welf. Sci. 6(1):1-23. http://dx.doi.org/10.1207/S15327604JAWS0601_01. PMid:12795855.

Kitala P., McDermott J., Kyule M., Gathuma J., Perry B. \& Wandeler A. 2001. Dog ecology and demography information to support the planning of rabies control in Machakos District, Kenya. Acta Tropica 78(3):217-230. http://dx.doi.org/10.1016/S0001-706X(01)00082-1. PMid:11311185.

Kongkaew W., Coleman P., Pfeiffer D.U., Antarasena C. \& Thiptara A. 2004. Vaccination coverage and epidemiological parameters of the owned-dog population in Thungsong District, Thailand. Prev. Vet. Med. 65(1/2):105115. http://dx.doi.org/10.1016/j.prevetmed.2004.05.009. PMid:15454330.

Lund E.M., Armstrong P.J., Kirk C.A., Kolar L.M. \& Klausner J.S. 1999. Health status and population characteristics of dog and cats examined at private veterinary practices in the United States. J. Am. Vet. Med. Assoc. 214(9):13361341. PMid:10319174.

Matter H.C., Wandeler A.I., Neuenschwander B.E., Harischandra L.P.A. \& Meslin F.X. 2000. Study of the dog population and the rabies control activities in the Mirigama area of Sri Lanka. Acta Tropica 75(1):95-108. http://dx.doi. org/10.1016/S0001-706X(99)00085-6. PMid:10708011.

Mialot J.P. 1988. Patologia da Reprodução dos Carnívoros Domésticos. Hora Veterinaria, Porto Alegre, p.87-95.

Michell A.R. 1999. Longevit of British breeds of dog and its relationships withsex, size, cardiovascular variables and disease. Vet. Rec. 145(22):625-629. http://dx.doi.org/10.1136/vr.145.22.625. PMid:10619607.

Mukherjee S. 2012. O Imperador de Todos os Males: uma biografia do câncer. Schwarcz, São Paulo, p.29.

Murray J.K., Browne W.J., Roberts M.A., Whitmarsh A. \& Gruffydd-Jones T.J. 2010. Number and ownership profiles of cats and dogs in the UK. Vet. Rec. 166(6):163-168. http://dx.doi.org/10.1136/vr.b4712. PMid:20139379.

Nunes C.M., Martines D.A., Fikaris S. \& Queiróz L.H. 1997. Avaliação da população canina da zona urbana do município de Araçatuba, São Paulo, 
SP, Brasil. Revta Saúde Pública 31(3):308-309. http://dx.doi.org/10.1590/ S0034-89101997000300013.

O’Neill D.G., Church D.B., McGreevy P.D., Thomson P.C. \& Brodbelt D.C. 2013. Longevity and mortality of owned dogs in England. Vet. J. 198(3):638-643. http://dx.doi.org/10.1016/j.tvjl.2013.09.020. PMid:24206631.

Olsen T.F. \& Allen A.L. 2000. Causes of sudden and unexpected death in dogs: a 10-year retrospective study. Can. Vet. J. 41(11):873-875. PMid:11126495.

Pal S.K. 2001. Population ecology of free-ranging urban dogs in West Bengal, India. Acta Theriol. 46:69-78. http://dx.doi.org/10.4098/AT.arch.01-8.

Patronek G.J., Waters D.J. \& Glickman T. 1997. Comparative longevity of pet dogs and humans: implications for gerontology research. J. Gerontol. A, Biol. Sci. Med. Sci. 52(3):171-178. http://dx.doi.org/10.1093/gerona/52A.3.B171. PMid:9158552.

Proschowsky H.F., Rugbjerg H. \& Ersboll A.K. 2003. Mortality of purebred and mixed-breed dogs in Denmark. Prev. Vet. Med. 58(1/2):63-74. http:// dx.doi.org/10.1016/S0167-5877(03)00010-2. PMid:12628771.
Pulczer A.S., Jones-Bitton A., Waltner-Toews D. \& Dewey C.E. 2013. Owned dog demography in Todos Santos Cuchumatán, Guatemala. Prev. Vet. Med. 108(2/3):209-217. http://dx.doi.org/10.1016/j.prevetmed.2012.07.012. PMid:22906504.

Ratsitorahina M., Rasambainarivo J.H., Raharimanana S., Rakotonandrasana H., Andriamiarisoa M., Rakalomanana F.A. \& Richard V. 2009. Dog ecology and demography in Antananarivo, 2007. BMC Vet. Res. 5(1):21. http:// dx.doi.org/10.1186/1746-6148-5-21. PMid:19486516.

Slater M.R., Di Nardo A., Pediconi O., Villa P.D., Candeloro L., Alessandrini B. \& Del Papa S. 2008. Cat and dog ownership and management patterns in central Italy. Prev. Vet. Med. 85(3/4):267-294. http://dx.doi.org/10.1016/j. prevetmed.2008.02.001. PMid:18374434.

Thrusfield M. 2004. Epidemiologia Veterinária. 2ª ed. Roca, São Paulo, p.57-60.

Withrow S.J., Vail D.M. \& Page R.L. 2013. Withrow and MacEwen's Small Animal Oncology. 5th ed. Elsevier, St Louis, p.136-138. 